\title{
La Biopsia de Endometrio en Infertilidad y como Indicadora de Insuficiencia Luteínica
}

\author{
(Estudio estadístico sobre 2.146 endometrios)
}

Dr. Gilberto Angel M.*

REVISION TEMAS

\section{RESUMEN:}

Entre 1950 y 1983, hemos tenido la oportunidad de analizar 2.146 endometrios, en pacientes que han consultado por infertilidad. El $90.17 \%$ fueron inaptos para la implantación. De estos un $25.28 \%$ correspondieron a ciclos anovulatorios y un $64.89 \%$ a ciclos ovulatorios pero con manifestaciones más o menos intensas de insuficiencia luteínica, que los incapacitan para tener éxito en la siembra embrionaria. Es un tejido con escasos elementos nutritivos y baja irrigación, a semejanza de un terreno arcilloso que es poco apto para una buena cosecha. En solo $9.8 \%$ de las pacientes, su causa de infertilidad no radicaba en su endometrio, pues estos eran normales en las dos capas funcionales. Llama poderosamente la atención que al endometrio se le considere en plano secundario en el proceso de implantación. En las publicaciones relacionadas con la fertilización in vitro, es profusa la literatura sobre

* Médico Patólogo-Clínico. Jefe Laboratorio Médico y Ginecológico.

Cali. ovulación, maduración, proliferación folicular, fertilización, implantación, etc. y sobre el terreno en donde se va a producir la siembra, es muy poca. Todos los endometrios reciben el mismo tratamiento, con dosis iguales de progesterona por el mismo tiempo, con resultados de implantaciones mínimas. No merecerá el endometrio una mayor atención?

La fertilización del óvulo bien sea en la trompa uterina de la madre o in vitro y la implantación posterior, natural o artificial, son procesos muy diferentes pero encaminados al mismo fin. Concebir un nuevo ser. Existe un proceso muy similar a cualquier cosecha y para obtener los frutos deseados, tenemos que contar con una buena semilla $y$ sembrarla en terreno adecuado.

Múltiples son los factores que se manifiestan en la pareja infértil, con ausencia de descendencia. Al corregir una o muchas de las causas que originan su infertilidad, se debe tener en cuenta el terreno donde se siembra, el abono y la irrigación. Al endometrio no se le debe considerar solamente como espejo infor- 
mativo que refleja si existió ovulación o no. Es un órgano que tiene una fisiología propia, cambiante cada día, capaz o incapaz de cumplir con la misión de desarrollar un nuevo fruto. Indudablemente que es el primer pilar en el terreno de la infertilidad. Pero en la práctica, muchas veces se le considera como un factor secundario y otras se le ignora.

En la fertilización in vitro, encontramos una nutrida y abundante literatura acerca de la ovulación, manera de producirla, proliferación folicular, maduración, fertilización, implantación, etc. y muy poco se encuentra sobre el endometrio. Yo me pregunto: ¿En las pocas implantaciones que tienen éxito sobre centenares realizadas, el endometrio no jugará algún papel? A priori y sin ninguna discriminación, en los centros donde se ocupan de la fertilización in vitro, tratan de corregir las supuestas fallas endometriales con dosis de progesterona, unificada para todos los casos y por el mismo tiempo.

En la estadística que presentames, se concluye que en un alto porcentaje $(74.71 \%$ ) los ciclos son bifásicos ovulatorios pero solo en $9.82 \%$ se puede verificar una implantación natural con éxito. Los otros presentan fallas en la producción o en la reacción endometrial a la luteína que a simple vista parece muy difícil tratar de corregir con una implantación en marcha.

\section{FISIOLOGIA DEL FOLICULO}

De los 400.000 folículos primordiales que tiene la niña al nacer, solo 420 van a proliferar $y$ algunos a madurar en los 35 años teóricos de fecundidad. La secreción hipofisiaria de $\mathrm{FSH}$, origina cada mes el crecimiento de uno de ellos el cual se transforma de primordial en vía de crecimiento. Se obtiene una proliferación de la granulosa y de la teca, hasta que la cavidad alcanza un diámetro de $6 \mathrm{mms}$. con un límite máximo de $7 \mathrm{mms}$.
De ahí en adelante, el óvulo se deñomina en maduración, se produce la meiosis, proceso esencial en la reducción cromatínica para poderse unir con el espermatozoide. La maduración puede ocurrir antes de abandonar el ovario o después, considerándose el licor folicular como un factor inhibidor de dicho proceso. Se interpreta lo anterior, como un factor de selección, es decir, que solo madura el ovocito fuerte $y$ sano que es capaz de contrarrestar dichos efectos inhibidores.

Después del estallido folicular por acción de la LH, se colapsan las paredes y la granulosa adquiere un aspecto plegado. De los vasos de la teca, avanza una proliferación muy abundante de capilares, que se introducen entre las células de la granulosa, disponiéndose las de la teca en campos o nidos. Desde este momento, se inicia la secreción interna que en forma paulatina y progresiva va a ejercer sus efectos sobre el endometrio, hasta el día 28 si no se produce implantación ovular o continuar en forma manifiesta si existe embarazo.

\section{FISIOLOGIA ENDOMETRIAL:}

Durante la menstruación, se descaman las capas superficiales del endometrio y se inicia el nuevo ciclo, a expensas de la capa basal regeneradora, que reposa sobre el miometrio. En los primeros 14 días del ciclo y por acción de los estrógenos, paulatinamente esta capa se va engrosando hasta llegar a tener al final de la mitad del ciclo, un espesor que varía entre 2 a $3 \mathrm{mms}$. Hasta este momento esta capa que se denomina funcional, está constituida por glándulas de luz estrecha, células cilíndricas altas y estroma citógeno, formando un aspecto uniforme de capa proliferativa. A las 36 horas de haber abandonado el óvulo al folículo, se aprecian notables cambios producidos por la progesterona, representados por vacuolas sub-nucleares que indican acumulación de glucógeno y empujan el 
núcleo de las células hacia la luz. Se ha iniciado el primer cambio en esta capa única, que de ahí en adelante se divide nítidamente en dos porciones. Una superficial que limita la luz de la cavidad uterina que se denomina capa compacta y otra inferior a ésta y que limita con la capa basal que se llama capa esponjosa. Cada una de ellas va a tener variaciones nítidas cada 24 horas, lo que permite individualizar el endometrio en cualquier momento del ciclo y fijarle el día a que corresponde. Las vacudas van aumentando hasta el día 20 en que aparece el primer cambio en la capa compacta, representado por el edema que justamente en este día y en el siguiente, alcanza su máxima expresión. Posteriormente, el estroma sufre modificaciones en sus células. Estas, de redondeadas, se hacen poliédricas y se cargan de glucógeno. A medida que va aumentando la progesterona, estas células son más nítidas, se forma la verdadera reacción pseudodecidual,que es más pronunciada alrededor de los vasos sanguíneos y ya con un nivel normal, se observan las arterias espirales, condicionadas exclusivamente a una buena concentración de progesterona $y$ que ejercen un gran papel en la placentación.

Si en la capa compacta son evidentes los cambios del estroma, estos también se aprecian en la esponjosa y al final del ciclo encontramos en esta, una verdadera esponja amortiguadora, formada por numerosas glándulas secretoras e insinuándose entre éllas una reacción pseudodecidual manifiesta.

La acción de la progesterona se va ejerciendo en forma paulatina $y$ las concentraciones séricas cada vez más altas son las encargadas de producir estos cambios tanto en la capa superficial compacta como en la intermedia esponjosa. Este detalle es muy importante de conocer para poder evaluar la insuficiencia lute ínica que tanta importancia tiene en la infertilidad. Las primeras y peque- ñas concentraciones solo modifican las glándulas manifestadas por vacuolas subnucleares y secreción en la luz glandular. Son un signo que nunca faltan en los ciclos ovulatorios. Los cambios en la compacta, representados por reacción pseudo-decidual requieren una dosis mayor de hormona y más aún la presencia de las arterias espirales. Estos datos nos indican en el estudio histológico al tener en cuenta el día en que ha sido tomada la biopsia, si la insuficiencia luteínica es leve, mediana o intensa, o si el ciclo es normal en todas sus manifestaciones. Cuando se observa una normalidad histológica en el día 27 del ciclo se puede afirmar que el terreno donde se va a verificar la siembra embrionaria, ofrece condiciones óptimas con energía inicialpara el embrión, representada por una buena producción de glucógeno en las glándulas $y$ en la reacción pseudodecidual. $Y$ factores benéficos para la placentación, representados por las arterias espirales, además del factor mecánico de ofrecer una verdadera esponja amortiguadora en la capa media.

\section{IMPLANTACION FISIOLOGICA:}

Sobre el día exactc en que normalmente se verifica la implantación, no existe un concepto unificado, predominando el del día 7 después de la ovulación cuando tanto el estroma como las glándulas han evolucionado favorablemente gracias a la acción de la progesterona. Cuando el óvulo fecundado llega al endometrio, lo hace en la etapa de blastocisto $y$ en este se distinguen dos componentes diferentes. Uno periférico denominado trofoblasto que va a originar la placenta con funciones específicas de nutrición, $y$ otra porción denominada embrioblasto de donde se va a originar el embrión. Cuando el blastocisto llega a la superficie del endometrio, penetra entre dos glándulas $y$ el trofoblasto digiere esta zona de mucosa, en forma agresiva, 
tal como lo hace el más maligno de los blastomas. Se denomina fase histiotrófica, por encontrarse en este momento con alimentación exclusivamente materna a costa de sus tejidos. Al hundirse el huevo queda incluido en el interior de la mucosa y digiere principalmente las células ricas en glucógeno. En la implantación fisiológica si no existe glucógeno, el embrión "perece por hambre" pues sus escasas reservas nutritivas se encuentran agotadas al final del largo viaje verificado durante 7 días a través de la trompa uterina. Inmediatamente se forma la cámara ovular. Algunos vasos se abren a dicha cámara y se inicia la nutrición del embrión, con la sangre materna. De ahí en adelante se establece la circulación placentaria y se cambia la fase histiotrófica por la hemotrófica.

Bien sea que consideremos la implantación fisiológica natural o la artificial in vitro, para que ésta tenga éxito se requiere un terreno abonado (glucógeno). Una irrigación abundante (arteriasespirales), todo esto inducido por la progesterona. Con ausencia de estos factores, si la implantación se verifica, fisiológicamente es muy transitoria.

En nuestro estudio estadístico que comprende un análisis sobre 2.146 endometrios, encontramos fuera de los ciclos anovulatorios, tres tipos de endometrios que reflejan ciclos ovulatorios pero inaptos para la implantación. Estos son: Bajos con hipoplasia difusa, asincrónicos y secreción disociada (insuficiencia lute ínica) que presentan las siguientes caracter ísticas:

\section{ENDOMETRIOS BAJOS CON HIPOPLASIA DIFUSA:}

Ocasionados bien sea por factores mecánicos que comprimen el miometrio (miomas) por factores constitucionales (hipotrofia genital), deficiencia estrogénica o hipofisiaria (FSH) las capas funcionales son tan delgadas que el trofo- blasto las digiere en su totalidad, penetra en el miometrio y se producen alteraciones histológicas importantes. Unas veces la cámara ovular se llena de sangre por la abundancia de capilares miometriales lesionados y se produce el micro-aborto que pasa inadvertido. Otras veces, puede originar la placenta ácreta o la previa, si se hace cerca del orificio interno cervical. De todas maneras, los endometrios bajos no ofrecen condiciones favorables para una buena implantación y posteriormente para una placentación normal.

\section{ENDOMETRIOS ASINCRONICOS:}

Su constitución histológica está retardada respecto al tiempo evolutivo, del ciclo ovárico. Por ejemplo endometrios tomados el día $27^{\circ}$ del ciclo, histológicamente se encuentran en los días $20^{\circ}-$ $22^{\circ}-23^{\circ}$, etc. Son ciclos ovulatorios que traducen insuficiencia lute ínica bien sea en la producción o en la recepción y en estas condiciones la implantación es imposible, pues es de suponer que en el día 21, no existe ningún cambio favorable para que esto suceda.

\section{ENDOMETRIOS CON MADURACION DISOCIADA O SECRECION DISOCIADA:}

Son ciclos ovulatorios en los que el endometrio siempre presenta glándulas secretoras, por reaccionar éstas con concentraciones bajas de progesterona, pero en el estroma no hay ningún cambio o son muy discretos que reflejen impregnación lute ínica. Entre las pacientes que consultaron por infertilidad, el $52.32 \%$ presentaron este tipo de alteración, encontrándose las restantes sintetizadas en el cuadro No.1. el cual nos refleja que en la infertilidad el endometrio juega como es de suponer, un gran papel y que se obtiene una utilidad muy relativa en el $64.89 \%$ de las pacientes analizadas reportando por el estudio histológico el simple dato de ciclo ovulatorio. Este so- 


\begin{tabular}{|c|c|c|}
\hline \multicolumn{3}{|l|}{ ENDOMETRIOS ANOVULATORIOS: } \\
\hline Proliferativos en mitad de dicha fase $\ldots \ldots$ & 12 & $0.55 \%$ \\
\hline Atróficos ................. & 27 & $1.25 \%$ \\
\hline \multirow[t]{2}{*}{ Proliferativos en final de dicha fase ..... } & 504 & $23.48 \%$ \\
\hline & $\overline{543}$ & $25.28 \%$ \\
\hline \multicolumn{3}{|l|}{ ENDOMETRIOS OVULATORIOS: } \\
\hline Bajos con Hipoplasia difusa . . . . . . . . . . & 34 & $1.58 \%$ \\
\hline Asincrónicos . . . . . . . . . . . & 236 & $10.99 \% c$ \\
\hline Secreción Disociada. . . . . . . . . . . & $\underline{1.123}$ & $52.32 \%$ \\
\hline CICLOS OVULATORIOS INAPTOS. . . . . & 1.393 & $64.89 \%$ \\
\hline ENDOMETRIOS NORMALES OVULATORIOS & 210 & $9.82 \%$ \\
\hline TOTALES $\ldots \ldots \ldots \ldots \ldots$ & 2.146 & $99.99 \%$ \\
\hline
\end{tabular}

lo es un primer paso eri una larga jornada $y$ un dato relativo si no se tiene en cuenta la fisiología endometrial.

Como concepto muy personal, en toda mujer infértil se debe conocer precisamente, si su endometrio ofrece las condiciones mínimas que permitan una implantación susceptible de evolucionar hasta el final. En caso contrario, corregir previamente con dosis e intensidad de tratamiento, según la modalidad histológica, sus alteraciones sin desestimar su fisiología, capacidad de reacción al estímulo lute ínico y los factores adversos o favorables que le presenta al embrión, para su completo desarrollo.

\section{DIA EN QUE DEBE TOMARSE LA BIOPSIA EN EL ESTUDIO DE INFERTILIDAD}

La biopsia de endometrio revela solamente un momento estático en la evolución de una fisiología permanente. El
Patólogo solo puede darle al Clínico información útil y eficiente, si conoce al menos la edad de la paciente y el día del ciclo en que se ha tomado dicha biopsia; datos que aunque elementales, a veces se carece de ellos. En infertilidad, es indispensable reconocer las capas compacta y esponjosa. A veces, endometrios tomados en la iniciación de la menstruación, ya han descamado su capa compacta y el diagnóstico entra más en el terreno de las probabilidades que en el de la realidad. El momento ideal está en los días 26 a 27 del ciclo, cuando se observan todos los cambios luteínicos. EI argumento de que es factible tomar la biopsia con un embarazo en evolución, es más teórico que real, pues justamente la paciente está consultando por su infertilidad crónica. Se debe tener en cuenta que hasta un $30 \%$ de las implantaciones son anormales y pasan a veces como pequeños retardos menstruales. 


\section{REFERENCIAS}

1. ACOSTA A: Editor del libro: Infertility Norfolk 1983.

2. ACKERMANS, Surgical Pathology $\left(E_{n}\right.$ dometrium 959-987) C.V. Mosly Co. Sixt. Edi. 1981.

3. AHUMADA J.C. DI PAOLA G, MURRAY G. SAMMARTINO R. Esterilidad conyugal. Edit. Panamericana Bs. As. 1964.

4. ANDERSON W. KISSANE J. Pathology (Endometrium 1.710-1.714) Mosby Company Sevent. Ed. 1977.

5. CHANNING C.P. Follicular fluid oocyte maturation inhibitor and follicular control of granulosa cell maturation. Infertility. 265-276. 1983.

6. DI PAOLA G. Fisiología de la fecunda ción. Esterilidad Conyugal 15-28 Edit Panamericana Bs. As. 1964.

7. EHRMANN R.L. In histology dating of the endometrium J. reprod. Med. 3: 179200, 1969.

8. FERENCZ A. BERTRAND G. GELFAND $M$. Proliferation kinetics of human endometrium during the normal menstrual cicle. Am. J. Obst. Gynec. 133: 859867. 1979.

9. GARCIA J,E. Monitoring the natural menstrual cycle for in vitro fertilization. Infertility Norfolk, 1-10. 1983.

10. GOOD R.G. MOYER D.L. Hormonal effectus on endometrium J. Fertil. Steril 19: 37-49, 1 os8.

11. HERTIG A,T. Gestational changes. Lab. Investig. 13: 1153-1191, 1964.

12. HERTIG A.T. A morphologic correlation of ovary, endometrium and corpora lutea during early pregnancy. Lab. Invest 13: 1153-1191, 1964. Ginecología y Obste tricia.

13. NOVACK E. WOODRUFF D. Histología del endometrio. 126-147, 1964. Edit. Alhambra Madrid. 1964.
14. NOYES R.W. HERTIG A.T. ROCK J. Dating the endometrium biopsy J. Fertil Steril. 1: 3-25, 1950.

15) NOYES R.W. Uniformity of secretory endometrium J. Fertil. Steril. 7: 103-109, 1956.

16) RUIZ V. BRUNO O. SILBERMAN J. Diagnóstico de la ovulación Esterilidad conyugal." 114-129. Edit. Panamer. Bs.As. 1964.

17. SANDOW B.A. Characteristics of human oocy tes asp irated for in vitro fertilization. Infertility. Norfolk. 143-147. 1983.

18. SAMmARTINO R. Patología del endometrio. Curso de perfeccionamiento celebrado en Mendoza 6-13 julio 1959. Publicado Bol. Cient. Soc. Mendoza IV. 553-669. 1960.

19. SAMMARTINO R. Endometrio normal y ciclo bifásico. Boletín Cient. Soc. Médica Mendoza IV : 564-571, 1960.

20. SAMMARTINO R. Ciclo fertil. Bol. Cient. Soc. Med. Mendoza IV: 572-576, 1960.

21. SAMMARTINO R. Variaciones individuales y patológicas del endometrio. Bol. Científico. Soc. Médica Mendoza IV: 577-587. 1960.

22. SAMMARTINO R. Alteraciones vascula res del endometrio. Bol. Cient. Soc. Méd. Mendoza; IV: 588-604, 1960.

23. SAMMARTINO R. Proliferaciones del tro foblasto. Bol. Cien. Soc. Méd. Mendoza: IV 642-648. 1960.

24. SAMMARTINO R. Trastornos de la anidación. Esterilidad conyugal (148-163). Edit. Panamericana. Bs. As. 1964.

25. STEVENSON C.S. Infertility. J. Fertil. Steril. 16: 208-222, 1965.

26. VEECK L. Fertilization and development Infertility-Norfolk (155-161). 1983. 